\title{
EQUIVALENCE OF ALGEBRAIC EXTENSIONS $\dagger$
}

\section{BY REINHOLD BAER}

The commutative fields $\ddagger K$ and $H$ are equivalent with regard to their common subfield $L$, if there exists an isomorphism between $K$ and $H$ which maps every element of $L$ upon itself. If $H$ and $K$ are equivalent with regard to $L$, then the same equations with coefficients in $L$ have solutions in $H$ and in $K$. It is the aim of this note to establish a criterion for the validity of the converse of the above proposition.

The field $F$ is completely algebraic with regard to its subfield $S$, if $F$ and $S$ satisfy:

(1) $F$ is algebraic with regard to $S$;

(2) if $\mathfrak{f}$ is an isomorphism of $S$ upon the subfield $S^{\prime}$ of the field $G^{\prime}$ such that every equation (with coefficients) in $S$ which has a solution in $F$ is mapped by $f$ upon an equation in $S^{\prime}$ which has a solution in $G^{\prime}$, then $\mathfrak{f}$ is induced by an isomorphism of $F$ upon a field $F^{\prime}$ between $S^{\prime}$ and $G^{\prime}\left(S^{\prime} \leqq F^{\prime} \leqq G^{\prime}\right)$.

E. Steinitz§ has proved that every simple algebraic extension $\|$ and every normal algebraic extension $\Psi$ is completely algebraic.

Lemma 1. If the algebraic extension $F$ of the field $S$ satisfies the condition (i) to every pair of fields $U$ and $V$ such that $S \leqq U \leqq V \leqq F$, $V$ finite with regard to $U$, there exists a field $W$ between $V$ and $F$ such that $W$ is finite and completely algebraic with regard to $U$, then $F$ is completely algebraic with regard to $S$.

Proof. There exists a chain of fields $F_{v}(v$ an ordinal number

$\dagger$ Presented to the Society, October 31, 1936.

$\ddagger$ Only commutative fields will be considered in this note.

\$ See E. Steinitz, Algebraische Theorie der Körper; Mit Erläuterungen und einem Anhang: Abriss der Galois-schen Theorie, neu herausgegeben von Reinhold Baer und Helmut Hasse, 1931.

$\| S$ is the simple algebraic extension of the field $F$, generated by the element $b$, if $b$ satisfies an algebraic equation with coefficients in $F$ and $S$ is a smallest field containing $F$ and $b$.

I $N$ is normal with regard to its subfield $S$ if every irreducible polynomial in $S$ which has zeros in $N$ is in $N$ a product of linear polynomials. 
taking all values between 0 and a certain ordinal number $k$ ) such that $F_{0}=S, F_{v+1}=F_{v}\left(e_{v}\right)$ is a simple (algebraic) extension of $F_{v}$ for $0 \leqq v \leqq k, F_{v}$ is the join of all the $F_{u}$ with $0 \leqq u<v$, if $v$ is a limit ordinal, $F_{k}=F$. This chain of fields $F_{v}$ satisfies in particular the relations $S \leqq F_{u} \leqq F_{v} \leqq F$, if $u<v$.

Assume now that $G^{\prime}$ is an algebraic extension of the field $S^{\prime}$ and that there exists an isomorphism of $S$ upon $S^{\prime}$ such that (I) every equation in $S$ with solutions in $F$ is mapped by upon an equation in $S^{\prime}$ with solutions in $G^{\prime}$.

There will now be constructed by complete (transfinite) induction a chain of fields $F_{v}^{\prime}$ and of isomorphisms $\mathfrak{f}_{v}$ of $F_{v}$ upon $F_{v}^{\prime}$ such that $S^{\prime} \leqq F_{u}^{\prime} \leqq F_{v}^{\prime} \leqq G^{\prime}$ for $u<v ; \mathfrak{f}_{0}=\mathbb{z}, \mathfrak{f}_{v}$ induces $\mathfrak{f}_{u}$ in $F_{u}$ for $u<v$; every $\mathfrak{f}_{v}$ satisfies (I). Since $F_{0}^{\prime}=S^{\prime}$ and $\mathfrak{f}_{0}=8$ is a suitable beginning for this construction, it may be assumed that $F_{v}^{\prime}, f_{v}$ have been defined for every $v$ with $0 \leqq v<u(\leqq k)$ and that they satisfy the above conditions.

\section{CASE 1. $u=w+1$ is not a limit ordinal.}

Denote by $g(x)$ the irreducible polynomial in $F_{w}$ whose zero is $e_{w}$. It is mapped by $f_{w}$ upon a certain polynomial $g^{*}(x)$ in $F_{w}^{\prime}$ and $g^{*}(x)$ has zeros in $G^{\prime}$, since $\mathrm{f}_{w}$ satisfies (I). Let $b_{1}, \cdots, b_{n}$ be the set of all the zeros of $g^{*}(x)$ in $G^{\prime} ; n$ is finite and positive. Since $g(x)$ is irreducible in $F_{w}, \mathfrak{f}_{w}$ is an isomorphism, it follows that $g^{*}(x)$ is irreducible in $F_{w}{ }^{\prime}$, and consequently there exists for every $i$ exactly one isomorphism $\mathfrak{h}_{i}$ which induces $\mathfrak{f}_{w}$ in $F_{w}$ and maps $F_{u}$ upon $F_{w}{ }^{\prime}\left(b_{i}\right)$ and $e_{u}$ upon $b_{i}$.

If $\mathfrak{h}_{i}$ does not satisfy (I), then there exists a polynomial $q_{i}(x)$ in $F_{u}$ which has zeros in $F$ such that the polynomial $q_{i}^{*}(x)$ upon which $q_{i}(x)$ is mapped by $\mathfrak{h}_{i}$ has no zero in $G^{\prime}$. Assume that none of the isomorphisms $\mathfrak{h}_{i}$ satisfies (I). The set of all the solutions in $F$ of the equation $g(x) q_{1}(x) \cdots q_{n}(x)=0$ generates a certain field $V$ between $F_{w}$ and $F$ which contains $F_{u}$ and is finite with regard to $F_{w}$. There exists therefore by condition (i) a field $W$ between $V$ and $F$ which is finite and completely algebraic with regard to $F_{w}$. Since the isomorphism $\mathfrak{f}_{w}$ of the subfield $F_{w}$ of $W$ satisfies (I), it follows that there exists an isomorphism $\mathfrak{h}$ of $W$ upon a field $W^{\prime}$ between $F_{w}^{\prime}$ and $G^{\prime}$ which induces $\mathfrak{f}_{w}$ in $F_{w}$. Since the isomorphisms $\mathfrak{h}_{1}, \cdots, \mathfrak{h}_{n}$ are all the possible isomorphisms of the subfield $F_{u}$ upon a field between $F_{w}^{\prime}$ and $G^{\prime}$ which induce $\mathfrak{f}_{w}$ in $F_{w}$, and since $F_{u}$ is a subfield of $W, \mathfrak{h}$ induces in $F_{u}$ exactly 
one of the isomorphisms $\mathfrak{h}_{i}$, say $\mathfrak{h}_{1}$. Since $q_{1}(x)$ has zeros in $W$, $q_{1}(x)$ is mapped by $\mathfrak{h}$ and by $\mathfrak{h}_{1}$ upon a polynomial in $F_{w}^{\prime}\left(b_{1}\right)$ which has zeros in $G^{\prime}$ in contradiction to the above assumption. Therefore at least one of the isomorphisms $\mathfrak{h}_{i}$, say $\mathfrak{h}_{n}$, satisfies (I) and it may be defined by $\mathfrak{f}_{u}=\mathfrak{h}_{n}, F_{u}^{\prime}=F_{w}{ }^{\prime}\left(b_{n}\right)$, and thus the chain of fields and isomorphisms has been prolongated in the required way.

\section{CASE 2. $u$ is a limit ordinal.}

Then $F_{u}$ is the join of the increasing chain of fields $F_{v}$ with $v<u$. If $F_{u}^{\prime}$ is defined as the join of the increasing chain of fields $F_{v}^{\prime}$ with $v<u$, then there exists one and only one isomorphism $\dagger \mathfrak{f}_{u}$ of $F_{u}$ upon $F_{u}^{\prime}$ which induces $\mathfrak{f}_{v}$ in $F_{v}$ for $v<u$. Since every polynomial in $F_{u}$ is contained in a certain $F_{v}$ with $v<u$, and since every $\mathfrak{f}_{v}$ with $v<u$ satisfies (I), it follows that also $\mathfrak{f}_{u}$ satisfies (I).

Thus it has been proved that fields $F_{u}^{\prime}$ and isomorphisms $\mathfrak{f}_{u}$, satisfying the above conditions, exist for every $u$ with $0 \leqq u \leqq k$. There exists therefore, in particular, an isomorphism $\mathfrak{f}_{k}$ of $F_{k}=F$ upon a field $F_{k}^{\prime}$ between $S^{\prime}$ and $G^{\prime}$ which induces the given isomorphism $\&$ in $S$, and therefore $F$ is completely algebraic with regard to $S$.

COROLlaRY 1. If $F$ is algebraic and separable $\ddagger$ with regard to its subfield $S$, then $F$ is completely algebraic with regard to $S$.

Proof. If $S \leqq U \leqq V \leqq F$, then $F$ and $V$ are separable with regard to $U . V$ is therefore a simple algebraic extension of $U$ if, and only if, $V$ is finite with regard to $U$. Since simple algebraic extensions are completely algebraic, it follows therefore that $F$ satisfies (i) with regard to $S$, and consequently Lemma $1 \mathrm{im}$ plies that $F$ is completely algebraic with regard to $S$.

THEOREM 1. Assume that $F$ is completely algebraic with regard to its subfield $S$, that $F^{\prime}$ is algebraic with regard to its subfield $S^{\prime}$, and that is an isomorphism of $S$ upon $S^{\prime}$. Then there exists an

$\dagger$ See E. Steinitz, loc. cit., Erläuterungen, pp. 17-18.

$\ddagger F$ is separable (and algebraic) with regard to its subfield $S$, if every element of $F$ is a solution of a separable irreducible equation in $S$. An irreducible equation $g(x)=0$ in $S$ is not separable if the characteristic of $S$ is a prime number $p$ and $g(x)=\sum_{i=0}^{n} s_{i} x^{p i}$. All the solutions of a separable equation are different. Every finite separable extension is simple. 
isomorphism of $F$ upon $F^{\prime}$ which induces in $S$ if, and only if, every polynomial $g(x)$ in $S$ has the same number of zeros in $F$ as the polynomial $g(x)=g^{*}(x)$ (upon which $g(x)$ is mapped by 8 ) has zeros in $F^{\prime}$.

Proof. Clearly it suffices to prove that the condition is sufficient. From the assumptions it follows that there exists an isomorphism $\mathfrak{f}$ of $F$ upon a field $F^{*}$ between $S^{\prime}$ and $F^{\prime}$ which induces $\mathcal{B}$ in $S$. Since $g(x)$ has as many zeros in $F$ as $g(x) \mathfrak{f}=g(x) \mathcal{B}$ has zeros in $F^{*}$ and in $F^{\prime}$, it follows that all the elements of $F^{\prime}$ which are algebraic with regard to $S^{\prime}$ are contained in $F^{*}$, and since $F^{\prime}$ is algebraic with regard to $S^{\prime}$, it follows that $F^{\prime}=F^{*}$, that is that $\mathbb{B}$ is induced by the isomorphism $\mathfrak{f}$ of $F$ upon $F^{\prime}$.

CoROLlARY 2. If $F$ is algebraic and separable with regard to its subfield $S$, and $F^{\prime}$ is algebraic with regard to its subfield $S^{\prime}$, then the isomorphism of $S$ upon $S^{\prime}$ is induced by an isomorphism of $F$ upon $F^{\prime}$ if, and only if, the polynomial $g(x)$ in $S$. has a zero in $F$ if, and only if, $g(x)$ has a zero in $F^{\prime}$.

Proof. It suffices to prove the sufficiency of the condition. Since an algebraic extension of a field is separable if, and only if, the solved irreducible equations of the subfield are separable, $\dagger$ $F^{\prime}$ is separable with regard to $S^{\prime}$. By Corollary $1, F$ and $F^{\prime}$ are completely algebraic with regard to $S$ and $S^{\prime}$, respectively. There exists therefore

an isomorphism $\mathfrak{f}$ of $F$ upon a field $G^{\prime}$ between $S^{\prime}$ and $F^{\prime}$ which induces $\&$ in $S$. an isomorphism $\mathfrak{g}$ of $F^{\prime}$ upon a field $G$ between $S$ and $F$ which induces $\mathfrak{g}^{-1}$ in $S^{\prime}$.

If the polynomial $g(x)$ in $S$ has $n$ zeros in $F$ and the polynomial $g(x)$ has $n^{\prime}$ zeros in $F^{\prime}$, then $n \leqq n^{\prime} \leqq n$, that is, $n=n^{\prime}$, since the zeros of $g(x)$ are mapped by $f$ upon zeros of $g(x) \&$ and the zeros of $g(x) \mathfrak{z}$ are mapped by $g$ upon zeros of $g(x)$. The isomorphism satisfies therefore the condition of Theorem 1 and is consequently induced by an isomorphism of $F$ upon $F^{\prime}$.

COROLlaRy 3. If $F$ and $G$ are algebraic with regard to their common subfield $S$, and if $F$ is separable with regard to $S$, then $F$ and $G$ are equivalent with regard to $S$ if, and only if, the same equations in $S$ have solutions in $F$ and in $G$. 
Suppose that $F$ is a field of characteristic $p \neq 0$ and that $K$ is a subfield of $F$. Then the element $x$ of $F$ is said to be a root with regard to $K$ if there exists an integer $n=n(x) \geqq 0$ such that $x^{p^{n}}$ is an element of $K$. The set $R(K<F)$ of all the elements in $F$ which are roots with regard to $K$ is a subfield of $F$, containing $K$. $\dagger$

Lemma 2. Suppose that $K_{i}$ is a subfield of the field $F_{i}$ of characteristic $p \neq 0$, and that there exists an isomorphism of $K_{1}$ upon $K_{2}$, satisfying the condition $(\mathrm{K})$ an equation with coefficients in $K_{1}$ has a solution in $F_{1}$ if, and only if, the (under $\mathrm{f}$ ) corresponding equation in $K_{2}$ has a solution in $F_{2}$. Then there exists one and only one isomorphism $\mathfrak{r}$ of $R\left(K_{1}<F_{1}\right)$ upon $R\left(K_{2}<F_{2}\right)$ which induces $\mathfrak{f}$ in $K_{1}$ and this isomorphism $\mathfrak{r}$ satisfies the condition ( $\left.\mathrm{R}\right)$ an equation with coefficients in $R\left(K_{1}<F_{1}\right)$ has a solution in $F_{1}$ if, and only if, the (under $\mathfrak{r})$ corresponding equation in $R\left(K_{2}<F_{2}\right)$ has a solution in $F_{2}$.

PRoof. If the element $b$ of $F_{i}$ is a root with regard to $K_{i}$, then there exists a smallest not negative integer $e=e(b)$ such that $b^{p^{(b)}}=b^{*}$ is an element of $K_{i}$. The polynomial $\ddagger f_{b}(x)=x^{p^{(b)}}-b^{*}$ is irreducible in $K_{i}$ and its only zero in $F_{i}$ is $b$, since $f_{b}(x)=(x-b)^{p^{(b)}}$ in $F_{i}$. Conversely, a polynomial of the form $x^{p^{i}}-c$ in $K_{i}$ has at most one zero in $F_{i}$.

If now $b$ is any element of $R\left(K_{1}<F_{1}\right)$, then it follows from these remarks and from condition $(\mathrm{K})$ that there exists exactly one zero of the polynomial $x^{p^{e(b)}}-b^{* *}$ in $F_{2}$, and this uniquely determined element of $F_{2}$ may be denoted by $b$ r. It follows from the mentioned properties of the fields $R\left(K_{i}<F_{i}\right)$ and from the equations $(x+y)^{p^{j}}=x^{p^{j}}+y^{p^{j}}$, that $\mathfrak{r}$ is an isomorphism of $R\left(K_{1}<F_{1}\right)$ upon $R\left(K_{2}<F_{2}\right)$ which induces $\mathfrak{l}$ in $K_{1}$, and that $\mathfrak{r}$ is the only isomorphism with this property.

From the formula which has just been mentioned it follows that there exists to every polynomial $f(x)$ in $R\left(K_{i}<F_{i}\right)$ a not negative integer $n$ such that $f(x)^{p^{n}}$ is a polynomial in $K_{i}$. The polynomial $f(x)=0$ has therefore a solution in $F_{i}$ if, and only if, the equation $f(x)^{p^{n}}=0$ (with coefficients in $K_{i}$ ) has a solution in $F_{i}$. Now $(\mathrm{R})$ is a consequence of $(\mathrm{K})$.

† See Steinitz, loc. cit., \$\$11-14.

‡ See Steinitz, loc. cit., $\$ \$ 11-14$. 
A consequence of this Lemma 2 and of Corollary 2 is the following corollary.

Corollary 4. Suppose that the field $F_{i}$ of characteristic $p \neq 0$ is algebraic with regard to its subfield $K$ and that $F_{1}$ is separable with regard to $R\left(K<F_{1}\right)$. Then $F_{1}$ and $F_{2}$ are equivalent extensions of their common subfield $K$ if, and only if, the same equations in $K$ have solutions in $F_{1}$ and in $F_{2}$.

REMARK. If $F$ is an algebraic extension of the field $K$ of characteristic $p \neq 0$, then it may happen that $F$ is not separable with regard to $R(K<F)$. $\dagger$ If $F$ is algebraic and normal with regard to $K$, then $F$ is always $\ddagger$ separable with regard to $R(K<F)$. Corollary 4 contains therefore the analogous proposition concerning normal algebraic extensions which has been mentioned in the beginning of this note.

The Institute for Advanced Study

$\dagger$ An example for this phenomenon may be found in R. Baer, Abbildungseigenschaften algebraischer Erweiterungen, Mathematische Zeitschrift, vol. 33 (1931), pp. 451-479, particularly pp. 471-472.

$\ddagger$ See Baer, loc. cit., Satz 14 on p. 471 ; for further criteria see Sätze 15 and 16. 